\title{
Experiencia en la administración de quimioterapia intraperitoneal en una unidad de diálisis peritoneal
}

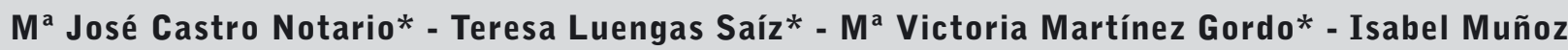
Gutiérrez* - Sara Rodríguez Rodríguez* - Gloria del Peso Gilsanz** - Marta Ossorio González** $M^{\text {a }}$ Auxiliadora Bajo Rubio**

*Enfermera

** Médico

Unidad de Diálisis Domiciliaria. Hospital Universitario La Paz. IDIPAZMadrid

\section{Sr. Director:}

El catéter de Tenckhoff es el más utilizado en el tratamiento con Diálisis Peritoneal de pacientes con Insuficiencia Renal ${ }^{1}$. Se ha utilizado como acceso a la cavidad peritoneal en otras patologías como: insuficiencia cardiaca co ngestiva ${ }^{2}$, pancreatitis necróticohemorrágicas ${ }^{3}$, ascitis malignas ${ }^{4}$ y para administración de quimioterapia intraperitoneal ${ }^{5}$.

La quimioterapia intraperitoneal se basa en la administración de fármacos antineoplásicos en la cavidad peritoneal, con el fin de tratar tumores que nacen o se extienden a dicha cavidad (Cáncer epitelial del ovario, Cáncer colorrectal, pseudomixoma peritoneal, cáncer gástrico...) Se utiliza principalmente como tratamiento coadyuvante tras la cirugía primaria, o como profilaxis en aquellos tumores con alto riesgo de recidiva peritoneal. Su objetivo es alcanzar concentraciones elevadas en peritoneo, con menor toxicidad sistémica, con el fin de eliminar focos micrometastásicos. Se utilizan diferentes agentes antineoplásicos como Mitomicina $C$, Cisplatino, Carboplatino, 0xaliplatino, Paclitaxel, Docetaxel, Mitoxantrone, Doxorubicina y 5 Fluoracilo, que debido a la barrera peritoneo-plasmática, permanecen largo tiempo dentro del abdomen antes de su absorción y eliminación, lo que los hace especialmente indicados en el tratamiento de la carcinomatosis peritoneal.

\begin{tabular}{|c|}
\hline Correspondencia: \\
M Jasé Castro Notario $^{\text {a Jarcial Lalanda 109 }}$ \\
28521 Rivas Vaciamadrid. Madrid \\
E-mail: mjcasnot@gmail.com \\
\hline
\end{tabular}

La carcinomatosis peritoneal es uno de los principales patrones de recaída tras la cirugía en los tumores de localización abdominal y pélvica. Se considera un signo de enfermedad generalizada, tratada de forma paliativa y de desenlace fatal, con una mediana de supervivencia de seis meses. La aplicación de una triple terapia combinada de cirugía citorreductora, junto con quimioterapia intraperitoneal perioperatoria, quimioterapia intraperitoneal intraoperatoria hipertérmica (QIIH), seguida de quimioterapia intraperitoneal postoperatoria precoz (QIPP), está permitiendo buenos resultados, incluso en ocasiones con intención curativa ${ }^{6}$.

Desde el punto de vista de enfermería la quimioterapia intraperitoneal intraoperatoria hipertérmica requiere de gran cantidad de cuidados debido tanto a la gran complejidad de la misma como a los efectos que este tipo de quimioterapia produce en el organismo ${ }^{7}$, pero no existe bibliografía acerca de los cuidados de enfermería que los pacientes requieren para seguir este tratamiento a más largo plazo.

En el año 2004, los servicios de Cirugía y de Oncología de nuestro Hospital, solicitaron la colaboración del equipo médico y de enfermería de la unidad de Diálisis Peritoneal, para iniciar el tratamiento con quimioterapia intraperitoneal en pacientes con carcinomatosis peritoneal utilizando para ello el catéter de Tenckhoff.

Establecimos conjuntamente un protocolo que incluía dos objetivos: el primero era asegurar la correcta administración de la quimioterapia y el segundo, relacionado con los cuidados del catéter peritoneal, era 
asegurar su adecuado funcionamiento y prevenir las complicaciones relacionadas con la técnica, fundamentalmente infecciones peritoneales e infecciones del catéter peritoneal.

El objetivo de nuestro trabajo fue evaluar los resultados obtenidos con la puesta en funcionamiento de dicho protocolo.

\section{Pacientes y métodos}

Desde febrero de 2004 hasta noviembre de 2009, hemos atendido a 10 pacientes de forma ambulatoria. El 60\% de los pacientes eran hombres. La edad media era de $46,77 \pm 11,98$ años, con un rango de 30 a 70 años.

Todos los pacientes tratados, estaban diagnosticados de carcinomatosis peritoneal. El $50 \%$ de los pacientes diagnosticado inicialmente de Adenocarcinoma colorectal, el $30 \%$ de Cistoadenoma apendicular, el $10 \%$ de Adenocarcinoma gástrico y el $10 \%$ de Pseudomixoma peritoneal $10 \%$.

La duración del tratamiento era de 6 meses. Al iniciar el protocolo, la pauta de administración de la quimioterapia era en ciclos mensuales de 5 días consecutivos, esta pauta se administró a cinco pacientes (50\%). Posteriormente los oncólogos cambiaron la forma administración, realizándose en ciclos quincenales de dos días consecutivos, de esta manera se administró al resto de los pacientes.

El catéter peritoneal utilizado fue de Tenckhoff recto con dos manguitos, implantado por los cirujanos en el transcurso de la cirugía citorreductora. Los cuidados del catéter, tempranos y a largo plazo, los realizábamos según nuestro protocolo habitual utilizado para los en diálisis peritoneal.

\section{El protocolo establecido consistía en:}

1. Realización, por parte del equipo de enfermería de la unidad, de los cuidados inmediatos posteriores a la implantación del catéter:

- Se realizaba la conexión de prolongador del catéter peritoneal (sistema de Baxter) según protocolo habitual.
- Se limpiaba el orificio con suero fisiológico y gasas estériles, se cubría con apósito estéril y se inmovilizaba para evitar tirones y traumatismos.

2. Administración de quimioterapia intraperitoneal: se inyectaba la medicación citostática (5-Fluouracilo) en $1000 \mathrm{ml}$ de Physioneal $^{\circledR}$ al 1,5\% y se infundía en la cavidad peritoneal, sin drenar previamente, mediante protocolo habitual de intercambio de diálisis peritoneal.

3. Realización de lavados peritoneales semanales: se realizaban en la unidad por parte del equipo de enfermería para evitar que el catéter se obstruyera. El protocolo de lavado era el habitual de nuestra unidad, se realizaba con Physioneal ${ }^{\circledR}$ al $1,5 \%$, dejando en el peritoneo $200-300 \mathrm{ml}$ de dicha solución a la que se añadía $5 \mathrm{ml}$ de heparina sódica al 1\%.

4. Entrenamiento del paciente para la realización de los cuidados del orificio en su domicilio una vez que fuera dado de alta:

- Se recomendaba la ducha según las costumbres del paciente.

- Se enseñaba a los pacientes las normas básicas de asepsia para la realización de los cuidados del catéter: uso de mascarilla, lavado y desinfección de manos.

- Se enseñaba la realización de la cura del orificio: limpieza con gasas estériles y con suero salino hipertónico y cubierto con apósito.

- Se enseñaba la correcta inmovilización del catéter y del prolongador.

- Se comentaba a los pacientes la posibilidad de desconexión accidental del prolongador o el tapón de este. Se les explicaba lo que debían hacer y se les enseñaba cómo poner un tapón nuevo.

- Se proveía a los pacientes del material necesario para la realización de los cuidados anteriormente citados: gasas, esparadrapo, suero salino hipertónico, tapones, mascarillas y desinfectante para las manos. 
Los datos se presentan los datos como valores medios \pm desviación estándar.

\section{Resultados}

Se han realizado 315 intercambios (185 para administrar medicación y 130 lavados) La duración media del catéter ha sido de 4,89 $\pm 2,6$ meses, con una rango de 0,93 a 10,03 meses. Seis pacientes $(60 \%)$ completaron el tratamiento prescrito. Tres pacientes lo abandonaron antes del primer mes debido un mal funcionamiento del catéter. Un paciente lo abandonó después de tres meses, por desplazamiento del catéter fuera del peritoneo y quedar alojado en el tejido subcutáneo.

El tiempo medio de supervivencia de los pacientes fue de 28,95 $\pm 23,30$ meses, con un rango 5,5 a 69,67 meses, sólo un paciente, el de mayor edad, falleció tras recibir un único ciclo.

No ha habido ningún episodio de peritonitis y tampoco hemos observado ninguna obstrucción del catéter. Sólo un paciente ha sufrido una infección aguda del orificio, causada por Stafilococo schleiferi, que se resolvió en una semana, tras tratamiento con Cloxacilina $500 \mathrm{mg}$ cada 8 horas por vía oral.

Sólo el paciente que sufrió el desplazamiento del catéter al tejido subcutáneo manifestó dolor intenso con la infusión del tratamiento. Los demás pacientes no manifestaron ninguna molestia durante el tratamiento y muy buena tolerancia.

\section{Conclusiones}

Dada la buena tolerancia del tratamiento que expresaron los pacientes, su capacidad para cuidarse el catéter y las escasas complicaciones observadas, especialmente las relacionadas con la técnica, nuestros datos sugieren que la administración ambulatoria de Quimioterapia Intraperitoneal realizada por un equipo de enfermería de diálisis peritoneal es sencilla y con una mínima incidencia de complicaciones.

\section{Bibliografía}

1. Kathuria P, Twardowski ZJ, and Nichols WK Peritoneal Dialysis Access and Exit-Site Care Including Surgical Aspects. En: Ramesh Khanna, Raymond Krediet, Eds. Nolph and Gokal's Textbook of Peritoneal Dialysis. Nueva York: Springer Science+Business Media; 2009. p 371-446.

2. Sánchez E, Rodriguez C, Ortega T, Díaz-Molina B, García-Cueto C. Papel de la diálisis peritoneal en el tratamiento de la insuficiencia cardíaca. Insuf Card 2010; (Vol 5) 3:105-112.

3. Beger HG, Büchler M, Bittner R, Oettinger W, Block $S$, Nevalainen T. Necrosectomy and postoperative local lavage in patients with necrotizing pancreatitis: results of a prospective clinical trial. World J Surg. 1988 Apr; 12(2):255-62.

4. Plancarte R, Guillén MR, Guajardo J, Mayer F. Ascitis en los pacientes oncológicos. Fisiopatogenia y opciones de tratamiento. Rev Soc Esp Dolor. 2004 11:156-162.

5. Yan TD, Welch $L$, Black $D$, et al. A systematic review on the efficacy of cytoreductive surgery combined with perioperative intraperitoneal chemotherapy for diffuse malignancy peritoneal mesothelioma. Ann Oncol. 2007;18:827-34.

6. Gómez Portilla A. Carcinomatosis peritoneal. Diez años aplicando la nueva triple terapia combinada. Experiencia personal. Cir Esp. 2007 Dec; 82(6): 346-351.

7. González Peña MG. Relevancia de los cuidados de enfermería en pacientes con carcinomatosis peritoneal, sometidos a citorreducción y quimioterapia intraoperatoria, intraperitoneal, hipertérmica (HIPEC). Enferm Comun 2009; 5(2). 